\title{
Outlining a Definition of Oral Health within the Study of Human Skeletal Remains
}

\author{
Marin A. Pilloud ${ }^{1 *}$ and James P. Fancher ${ }^{2}$ \\ ${ }^{1}$ Department of Anthropology, University of Nevada, Reno \\ ${ }^{2}$ Department of Anthropology, Texas State University, San Marcos
}

Keywords: periodontal disease, dental caries, hypercementosis, periapical lesions, calculus, antemortem tooth loss, linear enamel hypoplasia

\begin{abstract}
The term oral health is regularly used in bioarchaeological research to discuss a myriad of pathological conditions of the oral cavity. However, there is very little consensus on what conditions should be included in such a study, and some of the conditions are at odds with those in the clinical literature. In this manuscript, we outline the clinical definition of oral health and develop a strategy in which bioarchaeology can address this type of research. We argue that the terms dental disease and/or pathological conditions of the oral cavity should be used in lieu of oral health. Various conditions that can be included in such research are outlined. Finally, definitions, clinical etiologies, and recording schema for these conditions are discussed as relevant to bioarchaeological studies.
\end{abstract}

Recently the concept of "health" has been criticized in bioarchaeology (e.g., Reitsema \& McIlvaine, 2014). Defining health in past populations, or even among the recently deceased, can be difficult if not impossible as this concept incorporates somewhat unknowable factors about lifestyle and well-being. In response, the term "stress" was incorporated into research, which shifted the focus to conditions of disease or growth disruption. Yet, the etiology and interpretation of these markers of stress may not fully capture the experienced life of people in the past. For example, a study based on data from the Mexico Family Life Survey found that individuals with anemia were of various socioeconomic statuses and generally did not report being in poor health (Piperata et al., 2014). As skeletal signs of anemia are generally used in bioarchaeology as indicators of stress, this study of living individuals has highlighted the actual role of anemia in the lives of people currently experiencing it. Based on recent critiques, the incorporation of stress was cited as merely being a replacement for "poor health", while still failing to address the issues inherent in interpreting the levels of health and stress in past populations.

These critiques about quantifying general health and stress in bioarchaeology can be extended to the concept of oral health. A term that has been used in various contexts within the bioarchaeological literature to describe numerous conditions of the teeth and their surrounding bony structures. Despite its common use, there is currently no con- sensus on what should constitute oral health within the bioarchaeological record, and multiple indicators of stress, growth disruption, infection, oral pathology, and non-specific disease may be included. A more comprehensive discussion of oral health within the bioarchaeological record is warranted given inconsistencies in discussion of oral conditions and advances in the clinical literature.

In 2014, the American Dental Association (ADA) House of Delegates adopted the following definition of oral health as "a functional, structural, aesthetic, physiologic and psychosocial state of well-being... essential to an individual's general health and quality of life" (http:/ / www.ada.org/ en/about-the-ada/ada-positions-policies-andstatements/ada-policy-definition-of-oral-health). In 2016, the World Dental Federation (FDI, once called the Fédération Dentaire Internationale) adopted a new definition of oral health that echoes the sentiment of the ADA in that oral health is much more than the mere absence of disease. The FDI defines oral health as "the ability to speak, smile, smell, taste, touch, chew, swallow, and convey a range of emotions through facial expressions with confidence and without pain, discomfort, and

\footnotetext{
*Correspondence to:

Marin A. Pilloud

Department of Anthropology

University of Nevada, Reno

Reno, NV 89557

mpilloud@unr.edu
} 
disease of the craniofacial complex" (Glick et al., 2016:916). This work further emphasizes the interaction of disease status, physiological function, and psycho-social function along with determining and moderating factors that influence overall oral health (Glick et al., 2016).

From a research perspective, and in a clinical setting, it is critical to understand treatment outcomes and levels of oral health, as poor oral health can have further health and social implications. In recent clinical literature poor oral health has been linked to Type 2 diabetes (Leite et al., 2013), obesity (Östberg et al., 2012), and eating disorders (Johansson et al., 2012). Further, oral health has been related to school performance (Abanto et al., 2011; Jackson et al., 2011), quality of life, selfesteem (Bennadi \& Reddy, 2013; Gerritsen et al., 2010), and depression (Okoro et al., 2012). The concept of Oral Health-Related Quality of Life (OHRQoL) has grown over the last decade and is being recognized as a critical part of dental research and clinical dental practices (Sischo \& Broder, 2011). The OHRQoL is typically studied through questionnaires and serves as a means to quantify outcomes to better evaluate treatment in a clinical setting (Bennadi \& Reddy, 2013).

The definitions proposed by the ADA and FDI as well as the study of OHRQoL, raise questions about how bioarchaeologists currently use the term oral health, as it is not possible to understand the "psychosocial well-being" of past populations based only on the archaeological record. Further, the clinical research on oral health shows a disconnect with how the term is being used in bioarchaeological research. This paper proposes to address inconsistencies and misconceptions in studies of oral health on skeletal remains. We begin with a review of the current trends in the use of the term and end with a proposed outline of how the term can be used in research on skeletal remains.

\section{Current Use of Oral/Dental Health}

While the clinical definition of oral health includes conditions of the oral cavity that are detrimental to one's general quality of life, within bioarchaeological research this definition can vary widely. This variation is illustrated in a survey of articles published in the American Journal of Physical Anthropology, International Journal of Osteoarchaeology, and the International Journal of Paleopathology between 1977 and 2018. Those articles that use the term "dental health" or "oral health" in the title or abstract $(n=44)$ were searched to identify trends in the use of this term. Two of the articles that were recov- ered in the search were later found to not be related to oral health and were removed from further discussion.

In these articles, oral health was quantified through various skeletal indicators to include: periodontal disease, dental caries/carious lesions, linear enamel hypoplasia, crown variation, morphology, dental/occlusal wear, calculus, enamel hypoplasia, periapical defect/lesion/granuloma/ abscess, antemortem tooth loss, cleft palate, alveolar defects/lesions, chipping, dental tilting, hypercementosis, dens in dens, agenesis, and antemortem tooth loss. A word cloud was created to highlight the frequency of each type of skeletal indicator (Figure 1). By far the most commonly investigated conditions were dental caries, antemortem tooth loss, and abscesses.

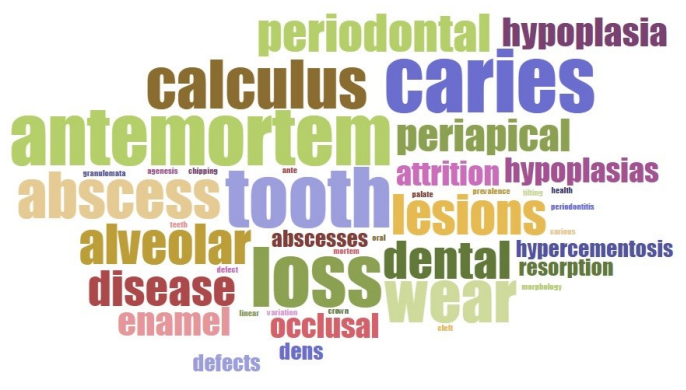

Figure 1. Word cloud highlighting use of terms in research on "oral/dental health".

Within this body of literature there is little consensus on what factors should be studied as part of oral health and may also employ varying definitions of these pathological conditions. However, these research foci are generally in line with the World Health Organization (WHO) definitions of dental disease, which include "dental cavities, periodontal (gum) disease, oral cancer, oral infectious diseases, trauma from injuries, and hereditary lesions" (http://www.who.int/mediacentre/ factsheets/fs318/en/). While these dental diseases may be relatively straightforward to diagnose in a clinical setting with a well-known patient history and clinical records, similar diagnoses in archaeological populations may be impossible to assess. We therefore dedicate the following section to outlining factors that can be used in defining diseases of the oral cavity in a bioarchaeological setting.

\section{Proposed Use}

We propose that based on recent critiques of the 
use of the term health, conditions of the oral cavity be termed dental disease or pathological conditions of the oral cavity. These terms shift the focus from the unknowable aspects of health (i.e., psychosocial well-being) to conditions that can be identified in the maxilla, mandible, and teeth with known etiologies. Below we outline which conditions can be used as part of this definition, conditions which are less applicable, and those that should not be included in studies of this nature.

\section{Conditions to include as dental disease/ pathological conditions of the oral cavity:}

Dental caries is a disease process characterized by dental hard tissue destruction of tooth enamel and dentin due to the bacterial fermentation of consumed carbohydrates. There can be many contributors to dental caries, to include diet, tooth morphology, calculus, age, sex, microbiology, and periodontal disease (Fakhruddin et al., 2018; Featherstone, 2008; Larsen, 2015; Young et al., 2015). A recent study of the human oral microbiome found that the progression of dental caries was related to a multiple bacterial species, not just Streptococcus mutans, as was previously thought. Further, individuals without carious lesions exhibited the presence of various other bacteria (e.g., genera of $A g$ gregatibacter and Rothia) that were found to impede the development of cariogenic bacteria (BeldaFerre et al., 2012).

In the clinical literature, much work has focused on the prevention of dental caries and increasing outcomes for patients. A Center for Disease Control and Prevention study on the presence of dental caries in the United States from 2005-2008 found that $75 \%$ of individuals had at least one dental restoration, and that $20 \%$ had untreated dental caries. Untreated dental caries differed significantly across socioeconomic status; although, younger individuals showed more equity in terms of dental restoration presence (Dye et al., 2012). These patterns are in line with recent studies in Canada and the United States that found a decline in socioeconomic inequalities in terms of oral health (Bernabé \& Marcenes, 2011; Elani et al., 2012).

Within bioarchaeological research, patterns in dental caries prevalence speak to disease loads and changing diets. The presence of carious lesions is also age dependent and will manifest differently in different teeth. There are various models for scoring dental caries in bioarchaeological research. In a clinical setting, rates of carious lesions are recorded using the DMFT method. In which the total number of diseased (D), missing due to disease (M), and filled teeth $(\mathrm{F})$, is divided by the total number of teeth (T). This system would clearly have limited utility in bioarchaeology due to the lack of filled teeth, teeth missing post-mortem, and the inability to determine the cause of antemortem tooth loss (Waldron, 2009).

For skeletal remains, the Moulage system could be employed, which is a series of 85 plaster models that illustrate varying degrees of carious decay in different locations (Hillson, 2001). However, these plaques were not made widely available outside of Scandinavia, and only a few photographs are available in published manuscripts (Lindström, 1940; Rönnholm et al., 1951), which makes their broad use impractical. Hillson (2001) proposed an alternate method for recording dental caries in which efforts are made to separate data in terms of tooth type, age cohort, sex, and lesion type and location. Such an approach can account for differential preservation of tooth types and age groups - both of which have an overall effect on dental caries prevalence calculations. While the method proposed by Hillson (2001) is by far the most nuanced and accounts for a range of biological variation, the most commonly employed method of dental caries recordation is that outlined in Buikstra and Ubelaker (1994). This method relies on a visual inspection of the dentition with recording of dental caries by tooth type and surfaces affected.

Periodontal disease is actually a cluster of inflammatory diseases that affect the periodontium (Langlais et al., 2017; Lindhe \& Lang, 2015). Clinically it is especially noted by the inflammatory status of gingiva and other soft tissues. The diseases are generally chronic and slow progressing in nature; although, more aggressive and acute forms do exist. The primary cause is a complex community of microbes that form a biofilm on tooth surfaces and interact with the host response systems to create an inflammatory response. There are many contributing factors that include local tooth anatomy, virulence of the biofilm, and systemic conditions that modify the host response. There are also various biological, social, and behavioral risk factors, which include: socioeconomic status, tobacco use, hormones, stress, excessive alcohol consumption, diabetes, obesity, osteoporosis, root abnormalities, enamel pearls, impacted third molars, and trauma, among many others (Jin et al., 2011). The WHO estimates that between 5 and $20 \%$ of adults globally have severe periodontitis (Jin et al., 2011). Periodontal disease remains a major cause of tooth loss in both developed and developing countries 
(Pihlström et al., 2005).

Disease limited to the gingiva often does not cause attachment loss and is usually reversible. Disease that affects the deeper structures of the periodontium lead to irreversible loss of connective tissue attachment and bone loss. Clinically, diagnosis is made based on a combination of measurable factors including pocket depth, clinical attachment loss, anatomical variations, tooth mobility, quantity and position of dental calculus, radiographic changes, and quantity of inflammation. The only factors that may reliably survive to the postmortem or bioarchaeological cases are quantity and position of dental calculus, attachment loss, and radiographic changes. A combination of these data is needed to assess the periodontal status of osseous specimens. Much evidence is currently available that links active periodontitis with numerous acute and chronic systemic diseases, including cardiovascular diseases, diabetes, pulmonary diseases, a cerebrovascular diseases (Albandar et al., 2018; Jepsen et al., 2018; Gerry J. Linden \& Herzberg, 2013; Gerard J. Linden et al., 2013)

There are multiple methods to score periodontal disease. In an epidemiological setting periodontal disease may be recorded using the Community Periodontal Index of Treatment Needs (CPITN), which requires a special probe to document the status of disease and treatment needs (Ainamo et al., 1982). (Comment: In fact, this system is somewhat flawed and not often used currently, but there is a long history in the literature). As there is no soft tissue in the bioarchaeological record, Karn et al. (1984) described a series of deformities of the alveolar process that include crater, moat, ramp, and plane to describe the disease process. Nearly a decade later, Kerr (1991) proposed an alternate scoring system in which the top of the interdental wall was described as flat/curved, porous, or with breakdown of the contour. Most recently, Waldron (2009) suggested the presence of periodontal disease should be recorded in individuals with $3 \mathrm{~mm}$ or greater distance between the cemento-enamel junction (CEJ) and the alveolar crest (AC) and recommended that the distance be documented with a periodontal probe. This is the method that most closely approximates the clinical gold standard for measuring the clinical attachment loss associated with periodontal disease, which is the diagnostic basis for defining periodontitis in humans (Eke et al., 2012; Holtfreter et al., 2015; Papapanou et al., 2018). However, when measuring skeletal remains it is also necessary to consider that the connective tissue component of the periodontal attachment apparatus is missing, and a $1 \mathrm{~mm}$ subtraction from the measured distance from the CEJ to the $\mathrm{AC}$ is necessary to allow for this missing portion of the biological width of tissue attachment (Gargiulo et al., 1961). The epidemiological threshold to define early periodontitis for human studies is $3 \mathrm{~mm}$ of attachment loss (Eke et al., 2018), which would be measured as $4 \mathrm{~mm}$ of distance from the CEJ to the AC. The clinical threshold that is often used is $2 \mathrm{~mm}$ of attachment loss (Papapanou et al., 2018) ( $3 \mathrm{~mm}$ measured from the CEJ to the AC), which encourages clinicians to diagnose and treat periodontitis at the earliest stages in order to prevent continued irreversible attachment loss.

Periapical lesions are related to pulpitis when an infection penetrates the pulp cavity. The term periapical lesion is a general term to describe a disturbance of the skeletal tissue around the apex of the tooth that may be related to a granuloma, cyst, or an abscess (Figure 2). The general term of 'periapical lesion' is preferred as the specific etiology is not possible to diagnose or differentiate without a soft tissue biopsy or a definitive patient history (Langlais et al., 2017). Even in a clinical setting these distinctions can be difficult to make (Stockdale \& Chandler, 1988), again underscoring the need to keep terminology general in bioarchaeological research. These lesions are merely recorded by location and as present or absent.

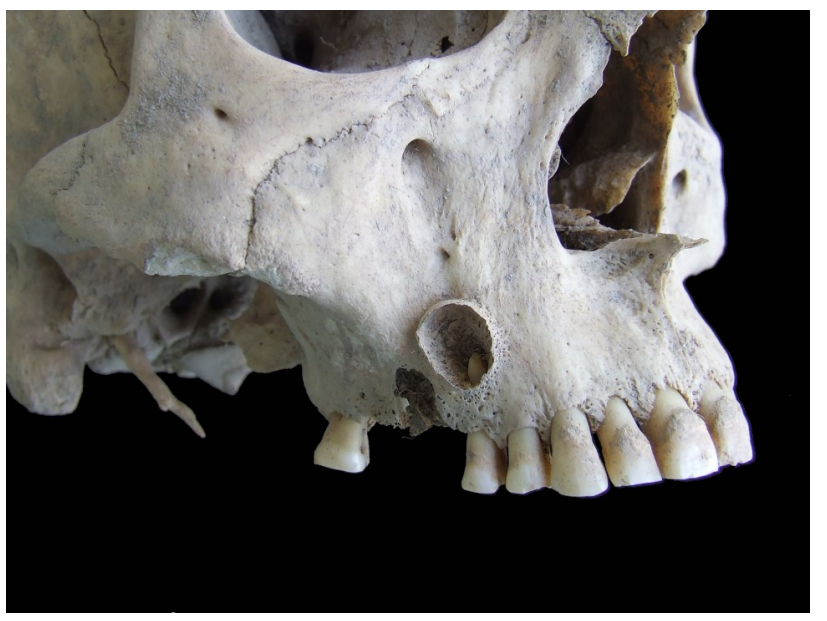

Figure 2. Example of a periapical lesion of the right maxillary fourth premolar.

Trauma to the oral cavity could be included under dental disease as it is a disorder of function. Further, it could affect the overall well-being of the individual and is included in clinical definitions of oral health. Traumata to the oral cavity could include acute trauma such as fractures to the mandi- 
ble, maxilla, or teeth; or, more long-term trauma such as damages due to bruxism, or issues with the temporomandibular joint.

Cancers of the oral cavity affecting bone are relatively rare in the bioarchaeological record, but could be included in a general study of oral disease according to the clinical definition of the WHO. The most common contemporary type of cancer in the oral cavity is squamous cell carcinoma, which may or may not leave a signature on bone. The incidence of oral cancer is also relatively low globally according to the Atlas of Oral Health (Beaglehole et al., 2009). Oral cancers also tend to have a greater prevalence in older humans, which should be considered in archaeological samples.

\section{Conditions to potentially include:}

Abscess/granuloma/cyst are terms that should generally be avoided individually as they require soft tissue and/or clinical evidence for a definitive diagnosis. As previously discussed, a more general term such as "periapical lesion" or "a certain anatomical lesion" is nearly always more accurate and includes the undetermined status of the lesion. In general, abscesses are acute lesions with purulent discharge, granulomas are chronic lesions with or without discharge, and cysts are epithelial-lined benign neoplasms. These all may look the same radiographically or as osseous lesions.

Calculus is a mineralized biofilm on the surface of teeth. The accumulation and composition of calculus can be studied to understand the oral microbiome, or may be measured in relation to other disease parameters (e.g., periodontal disease). However, the exact process of mineralization is not fully understood (Warinner et al., 2015). Therefore, calculus is thought to be more a product of disease processes rather than the cause of disease, and may be best included as part of pathological conditions of the oral cavity as opposed to dental diseases or can be included in a diagnosis of periodontal disease. In bioarchaeology, the presence of calculus is typically recorded according to the scheme developed by Brothwell (1981) in which calculus presence is scored on a scale from 1 to 3 of increasing severity.

Hypercementosis is the excessive build-up of cementum with unknown etiology (Corruccini et al., 1987). Although, it has been suggested to be linked to genes, Paget's disease, rheumatoid arthritis, thyroid goiter, acromegaly, and rheumatic fever
(Mohan, 2014). As the etiology is unknown, it is not appropriate to include it as part of studies focused on dental disease. However, it may be recorded as a dental anomaly or in the differential diagnosis of other, potentially related, conditions.

Antemortem tooth loss (AMTL) should be used cautiously as there are many causes of this condition, some of which may not be related to disease (e.g., dental ablation and trauma). Care must also be taken in cases where dental agenesis may be a factor (e.g., third molars, upper lateral incisors, or lower third premolars). It is critical that radiographs are taken in ambiguous cases to ensure impaction is not a factor. Although in the clinical literature, tooth loss is largely related to dental disease, predominantly periodontal disease and dental caries (Gerritsen et al., 2010). Antemortem tooth loss can more definitively be assigned in studies of dental disease in cases where there is large gap, reactive bone, a healed alveolar ridge with a deficient volume of bone, or it is a tooth that is not typically missing due to agenesis, trauma or ablation (Figure 3). In cases of uncertainty, antemortem tooth loss should not be recorded.

\section{Conditions to limit or not include:}

Enamel hypoplasia is a disruption in enamel secretion during dental development (Goodman \& Rose, 1990). These enamel defects can manifest as pits, furrows, or plane defects (Hillson \& Bond, 1997). Enamel hypoplastic defects are linked to episodic childhood stress, such as malnutrition and fevers (Hillson, 1996). As such, they are an indication of general stress, not specific to the oral cavity, and are therefore not applicable to a study cen-

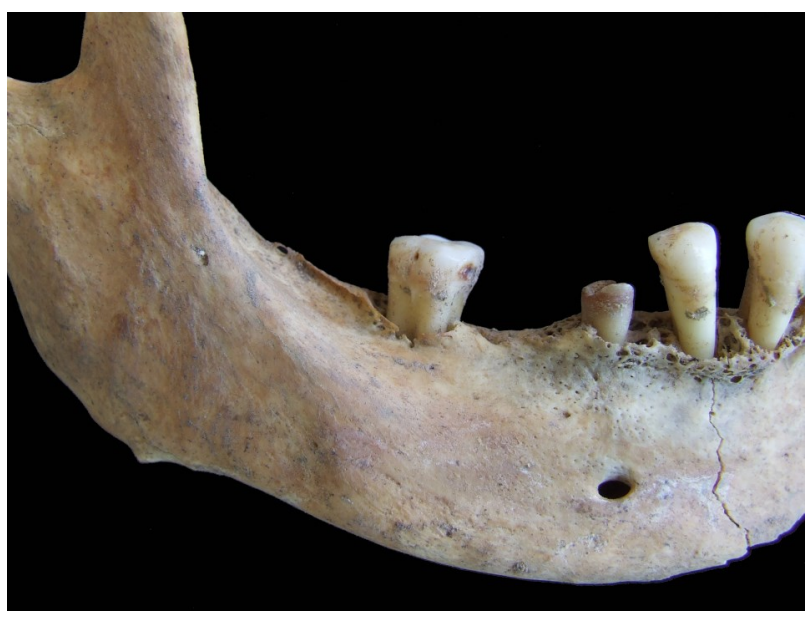

Figure 3. Example of antemortem tooth loss with reactive bone and a large gap on the right mandible. 
tered on dental disease.

Occlusal wear is a reduction in the dental hard tissues related to traumatic injuries to teeth caused by abrasion, attrition, and/or erosion (Ibsen \& Phelan, 2018; Langlais et al., 2017). Although these injuries may lead to reduced function of the dentition due to loss or alteration of dental hard tissues, the occlusal wear itself is not a disease process, but is the result of either (1) wearing away of tooth structure from a repetitive mechanical habit (abrasion), (2) wearing away of tooth structure due to tooth-tooth contact (attrition), or (3) loss of tooth structure resulting from chemical action not of bacterial origin (erosion). Unless the processes or results of occlusal wear can be specifically identified as pathological, such as there is associated pulp exposure with infection, general wear is not an indicator of dental disease. It is likely more related to the time span of life (often expressed as age), consumed foods, the use of teeth as tools, sex, bite force, malocclusion, environmental or salivary factors (Dahl et al., 1993).

Tooth size/morphology/agenesis are all conditions that are considered highly heritable and should be studied as separate conditions. Tooth size may be related to non-specific indicators of childhood or maternal stress (Pilloud \& Kenyhercz, 2016), and therefore is not related to studies of dental disease. Moreover, there are no good data to show that morphology or dental agenesis are related to stress or a disease process.

Chipping is related to microtrauma and therefore a reflection of biting force and potentially diet and tool use (Scott \& Winn, 2011). Chipping also may be due to blunt force, or an eventual result of wear. Unless the chipping leads to pulp exposure and infection, or possibly radiographic signs of secondary dentin formation as a result of the trauma, it is not a disease process on its own.

Cleft palate is a congenital defect that is also not related to any active disease process, and it should be considered separately. Even though it may affect overall function of the oral cavity, there is much variation in expression and severity and due to its relative rarity in the archaeological record, it should be considered independently in studies of pathological conditions of the oral cavity.

\section{Discussion and Conclusions}

The study of pathological conditions of the oral cavity in the past are an important aspect of archaeological research. These conditions can inform our understanding of diet, disease loads, activity, and genetic composition of past populations. We argue that this research is an important endeavor that is only lacking in a level of clinical standardization and appreciation. Measures of dental disease in the archaeological record that can conform to current measures of dental disease will improve the relevance and understanding of disease processes that can be related over the continuum of human history. This manuscript is meant to start the discussion of standardizing research on dental disease with an emphasis on clinical research. This work is not meant to be the definitive word on bioarchaeological research on oral health or dental disease; instead, we hope to generate a discussion on this research and work towards an integrated approach that fully intertwines bioarchaeological research within a clinical reality that embraces the accurate use of terms.

\section{Acknowledgments}

We thank all the participants who were part of the original symposium on oral health at the American Association of Physical Anthropology annual meetings in Austin, Texas in 2018. We also thank the Dental Anthropology Association for sponsoring the symposium from which this paper stems. And, we thank Başak Boz for reviewing this paper.

\section{REFERENCES}

Abanto, J., Carvalho, T. S., Mendes, F. M., Wanderley, M. T., Bönecker, M., \& Raggio, D. P. (2011). Impact of oral diseases and disorders on oral health-related quality of life of preschool children. Community Dentistry and Oral Epidemiolo$g y, 39(2), 105-114$.

Ainamo, J., Barmes, D., Beagrie, G., Cutress, T., Martin, J., \& Sardo-Infirri, J. (1982). Development of the World Health Organization (WHO) community periodontal index of treatment needs (CIPTN). International Dental Journal, 32(3), 281-291.

Albandar, J. M., Susin, C., \& Hughes, F. J. (2018). Manifestations of systemic diseases and conditions that affect the periodontal attachment apparatus: Case definitions and diagnostic considerations. Journal of Periodontology, 89(S1), S183-S203.

Beaglehole, R., Benzian, H., Crail, J., \& Mackay, J. (2009). The Oral Health Atlas: Mapping a neglect- 
ed global health issue. Geneva: FDI World Dental Federation.

Belda-Ferre, P., Alcaraz, L. D., Cabrera-Rubio, R., Romero, H., Simón-Soro, A., Pignatelli, M., \& Mira, A. (2012). The oral metagenome in health and disease. The ISME journal, 6(1), 46-56.

Bennadi, D., \& Reddy, C. V. K. (2013). Oral health related quality of life. Journal of International Society of Preventive \& Community Dentistry, 3 (1), 1-6.

Bernabé, E., \& Marcenes, W. (2011). Income inequality and tooth loss in the United States. Journal of Dental Research, 90(6), 724-729.

Brothwell, D. R. (1981). Digging up bones. Ithaca, New York: Cornell University Press.

Buikstra, J. E., \& Ubelaker, D. H. (Eds.). (1994). Standards for data collection from human skeletal remains. Fayetteville, Arkansas: Arkansas Archeological Survey Research Series No. 44.

Corruccini, R. S., Jacobi, K. P., Handler, J. S., \& Aufderheide, A. C. (1987). Implications of tooth root hypercementosis in a Barbados slave skeletal collection. American Journal of Physical Anthropology, 74(2), 179-184.

Dahl, B. L., Carlsson, G. E., \& Ekfeldt, A. (1993). Occlusal wear of teeth and restorative materials: a review of classification, etiology, mechanisms of wear, and some aspects of restorative procedures. Acta Odontologica Scandinavica, 51 (5), 299-311.

Dye, B. A., Li, X., \& Beltrán-Aguilar, E. D. (2012). Selected oral health indicators in the United States, 2005-2008: US Department of Health and Human Services, Centers for Disease Control and Prevention.

Eke, P. I., Page, R. C., Wei, L., Thornton-Evans, G., \& Genco, R. J. (2012). Update of the case definitions for population-based surveillance of periodontitis. Journal of Periodontology, 83(12), 14491454.

Eke, P. I., Thornton-Evans, G. O., Wei, L., Borgnakke, W. S., Dye, B. A., \& Genco, R. J. (2018). Periodontitis in US Adults: National Health and Nutrition Examination Survey 2009 -2014. The Journal of the American Dental Association, 149(7), 576-588.e576.

Elani, H., Harper, S., Allison, P., Bedos, C., \& Kaufman, J. (2012). Socio-economic inequalities and oral health in Canada and the United States. Journal of Dental Research, 91(9), 865-870.

Fakhruddin, K. S., Ngo, H. C., \& Samaranayake, L. P. (2018). Cariogenic microbiome and microbiota of the early primary dentition: A contemporary overview. Oral Diseases, 25(4), 982-995.
Featherstone, J. (2008). Dental caries: a dynamic disease process. Australian Dental Journal, 53(3), 286-291.

Gargiulo, A. W., Wentz, F. M., \& Orban, B. (1961). Dimensions and Relations of the Dentogingival Junction in Humans. Journal of Periodontology, 32(3), 261-267.

Gerritsen, A. E., Allen, P. F., Witter, D. J., Bronkhorst, E. M., \& Creugers, N. H. J. (2010). Tooth loss and oral health-related quality of life: a systematic review and meta-analysis. Health and Quality of Life Outcomes, 8(1), 126.

Glick, M., Williams, D. M., Kleinman, D. V., Vujicic, M., Watt, R. G., \& Weyant, R. J. (2016). A new definition for oral health developed by the FDI; World Dental Federation opens the door to a universal definition of oral health. The Journal of the American Dental Association, 147(12), 915-917.

Goodman, A. H., \& Rose, J. C. (1990). Assessment of systemic physiological perturbations from dental enamel hypoplasias and associated histological structures. Yearbook of Physical Anthropology, 33(59-110).

Hillson, S. (1996). Dental Anthropology. Cambridge: Cambridge University Press.

Hillson, S. (2001). Recording dental caries in archaeological human remains. International Journal of Osteoarchaeology, 11(4), 249-289.

Hillson, S., \& Bond, S. (1997). Relationship of enamel hypoplasia to the pattern of tooth crown growth: a discussion. American Journal of Physical Anthropology, 104, 89-103.

Holtfreter, B., Albandar, J. M., Dietrich, T., Dye, B. A., Eaton, K. A., Eke, P. I., Papapanou, P. N., \& Kocher, T. (2015). Standards for reporting chronic periodontitis prevalence and severity in epidemiologic studies. Journal of Clinical Periodontology, 42(5), 407-412.

Ibsen, O. A. C., \& Phelan, J. A. (2018). Oral pathology for the dental hygienist. St Louis, MO: Elsevier.

Jackson, S. L., Vann, W. F., Jr., Kotch, J. B., Pahel, B. T., \& Lee, J. Y. (2011). Impact of poor oral health on children's school attendance and performance. American Journal of Public Health, 101 (10), 1900-1906.

Jepsen, S., Caton, J. G., Albandar, J. M., Bissada, N. F., Bouchard, P., Cortellini, P., Demirel, K., Sanctis, M., Ercoli, C., Fan, J., Geurs, N. C., Hughes, F. J., Jin, L., Kantarci, A., Lalla, E., Madianos, P. N., Matthews, D., McGuire, M. K., Mills, M. P., Preshaw, P. M., Reynolds, M. A., Sculean, A., Susin, C., West, N. X., \& Yama- 
zaki, K. (2018). Periodontal manifestations of systemic diseases and developmental and acquired conditions: Consensus report of workgroup 3 of the 2017 World Workshop on the Classification of Periodontal and PeriImplant Diseases and Conditions. Journal of Periodontology, 89(S1), S237-S248.

Jin, L., Armitage, G., Klinge, B., Lang, N., Tonetti, M., \& Williams, R. (2011). Global oral health inequalities: task group - periodontal disease. Advances in Dental Research, 23(2), 221-226.

Johansson, A. K., Norring, C., Unell, L., \& Johansson, A. (2012). Eating disorders and oral health: a matched case-control study. European Journal of Oral Sciences, 120(1), 61-68.

Karn, K. W., Shockett, H. P., Moffitt, W. C., \& Gray, J. L. (1984). Topographic Classification of Deformities of the Alveolar Process. Journal of Periodontology, 55(6), 336-340.

Kerr, N. W. (1991). Prevalence and natural history of periodontal disease in Scotland - The mediaeval period (900-1600 A. D.). Journal of Periodontal Research, 26(4), 346-354.

Langlais, R. P., Miller, C. S., \& Gehrig, J. S. (2017). Color atlas of common oral diseases, Fifth Edition. Philadelphia: Wolters Kluwer.

Larsen, C. S. (2015). Bioarchaeology: Interpreting behavior from the human skeleton. Cambridge: Cambridge University Press.

Leite, R. S., Marlow, N. M., Fernandes, J. K., \& Hermayer, K. (2013). Oral Health and Type 2 Diabetes. The American Journal of the Medical Sciences, 345(4), 271-273.

Linden, G. J., \& Herzberg, M. C. (2013). Periodontitis and systemic diseases: a record of discussions of working group 4 of the Joint EFP/ AAP Workshop on Periodontitis and Systemic Diseases. Journal of Periodontology, 84(4-s), S20S23.

Linden, G. J., Lyons, A., \& Scannapieco, F. A. (2013). Periodontal systemic associations: review of the evidence. Journal of Periodontology, 84(4-s), S8-S19.

Lindhe, J., \& Lang, N. P. (Eds.). (2015). Clinical Periodontology and Implant Dentistry, Sixth Edition. West Suffix, UK: John Wiley \& Sons.

Lindström, P. (1940). Preliminärt förslag till en standard. Odontologisk Tidskrift, 48, 91.

Mohan, B. (2014). Hypercementosis and concrescence of maxillary second molar with third molar: a case report and review of literature. Oral Health Dental Management, 13(2), 558-561.

Okoro, C. A., Strine, T. W., Eke, P. I., Dhingra, S. S., \& Balluz, L. S. (2012). The association between depression and anxiety and use of oral health services and tooth loss. Community Dentistry and Oral Epidemiology, 40(2), 134-144.

Östberg, A.-L., Bengtsson, C., Lissner, L., \& Hakeberg, M. (2012). Oral health and obesity indicators. BMC Oral Health, 12(1), 50.

Papapanou, P., Sanz, M., Buduneli, N., Dietrich, T., Feres, M., Fine, D. H., Flemmig, T., Garcia, R., Giannobile, W., Graziani, F., Greenwell, H., Herrera, D., Kao, R., Kebschull, M., Kinane, D., Kirkwood, K., Kocher, T., Kornman, K., Kumar, P., Loos, B., Machtei, E., Meng, H., Mombelli, A., Needleman, I., Offenbacher, S., Seymour, G., Teles, R., \& Tonetti, M. (2018). Periodontitis: Consensus report of workgroup 2 of the 2017 World Workshop on the Classification of Periodontal and Peri-Implant Diseases and Conditions. Journal of Periodontology, 89(S1), S173-S182.

Pihlström, B., Michalowicz, B., \& Johnson, N. (2005). Periodontal diseases. Lancet Infectious Diseases, 366, 1809-1820.

Pilloud, M. A., \& Kenyhercz, M. W. (2016). Dental metrics in biodistance analysis. In M. A. Pilloud \& J. T. Hefner (Eds.), Biological Distance Analysis: Forensic and Bioarchaeological Perspectives (pp. 135-155). San Diego: Academic Press.

Piperata, B. A., Hubbe, M., \& Schmeer, K. K. (2014). Intra-population variation in anemia status and its relationship to economic status and self -perceived health in the Mexican Family Life Survey: Implications for bioarchaeology. American Journal of Physical Anthropology, 155(2), 210220.

Reitsema, L. J., \& Mcllvaine, B. K. (2014). Reconciling "stress" and "health" in physical anthropology: What can bioarchaeologists learn from the other subdisciplines? American Journal of Physical Anthropology, 155(2), 181-185.

Rönnholm, E., Markén, K.-E., \& Arwill, T. (1951). Record systems for dental caries and other conditions of the teeth and surrounding tissues. Odontologisk Tidskrift, 59, 34-56.

Scott, G. R., \& Winn, J. R. (2011). Dental chipping: contrasting patterns of microtrauma in Inuit and European populations. International Journal of Osteoarchaeology, 21(6), 723-731.

Sischo, L., \& Broder, H. (2011). Oral health-related quality of life: what, why, how, and future implications. Journal of Dental Research, 90(11), 1264-1270.

Stockdale, C., \& Chandler, N. (1988). The nature of the periapical lesion - a review of 1108 cases. Journal of Dentistry, 16(3), 123-129. 
Waldron, T. (2009). Paleopathology. Cambridge: Cambridge University Press.

Warinner, C., Speller, C., \& Collins, M. J. (2015). A new era in palaeomicrobiology: prospects for ancient dental calculus as a long-term record of the human oral microbiome. Philosophical Transactions of the Royal Society B, 370(1660), 20130376.

Young, D. A., Nový, B. B., Zeller, G. G., Hale, R., Hart, T. C., Truelove, E. L., Ekstrand, K. R., Featherstone, J. D. B., Fontana, M., Ismail, A., Kuehne, J., Longbottom, C., Pitts, N., Sarrett, D. C., Wright, T., Mark, A. M., \& BeltranAguilar, E. (2015). The American Dental Association Caries Classification System for Clinical Practice. The Journal of the American Dental Association, 146(2), 79-86. 